\title{
The Changing Role of French Local Government
}

\author{
Emmanuel Négrier \\ for West European Politics \\ Special Issue : « The Changing French Political System » \\ Vol.22 n4, Frank Cass, Londres, octobre 1999 pp.120-140
}

Analyser le gouvernement local en France, c'est s'interroger simultanément sur les changements qui l'ont affecté, notamment depuis la décentralisation du début des années 1980, et sur l'évolution des concepts et outils d'analyse qui visent à en comprendre les aspects essentiels. La notion de gouvernement local elle-même a longtemps fait l'objet d'une réticence, à la fois politique et académique en France. D'une part, elle était contradictoire avec une tradition française, d'inspiration jacobine, de méfiance à l'égard des pouvoirs locaux. Jusqu'à la période de décentralisation du début des années 1980, parler de gouvernement était largement excessif compte tenu du pouvoir que l'Etat central exerçait, sur le territoire, en termes de contrôle et d'exécution des affaires publiques. L'autonomie qui était l'une des conditions de l'expression anglaise de local government, était trop limitée pour donner lieu à de vrais gouvernements territoriaux ou à engendrer de véritables politiques locales (Rangeon 1996, Schmidt 1990). Pourtant, dès cette période, l'idée d'un pouvoir central de l'Etat s'exerçant sans partage ni inflexion locale était contredite par les faits et l'observation sociologique (Worms 1966, Cole \& John 1997a). Parallèlement à l'expression officielle du centralisme, se pratiquait un système de négociation informelle entre l'Etat et les notables locaux. Ce n'est donc pas un hasard si, à la place du gouvernement local, l'analyse politique française ait davantage raisonné en termes de système politico-administratif local (Grémion 1976). Celui-ci a en effet l'avantage de mettre l'accent, au delà du cadre réglementaire, sur la sociologie des relations entre centre et périphérie, et sur la reproduction, dans le temps et dans l'espace, de systèmes de négociation permanente de la règle, de son application, et des politiques publiques qui affectent le territoire. Au travers de cette analyse, les élus (officiellement faibles) et les bureaucrates (les représentants locaux de 
l'Etat, officiellement puissants), loin d'être séparables, sont en fait placés dans une situation d'interdépendance stratégique qui fait système. L'Etat, par le biais de ses agents, et notamment le préfet, qui occupe une place centrale dans le dispositif social et politique, dispose des instruments d'action mais doit en négocier la «faisabilité territoriale» avec des élus qui, démunis de ressources juridiques et économiques, sont la clef de l'acceptabilité des politiques centrales et en négocient l'adaptation locale, les inflexions et exceptions. Ce système reposait sur un échange politique simple : il garantissait à l'Etat le maintien et l'extension du consensus social et politique ; il permettait aux élus d'obtenir de l'Etat un élargissement des ressources locales et une légitimation auprès des citoyens. Un bon élu était celui qui disposait d'un accès facilité à l'administration ; un grand élu était celui qui parvenait à élargir et à diversifier la palette d'accès à l'administration. Ce système permettait d'établir une certaine hiérarchisation des problèmes politiques. Elle se faisait notamment en fonction de l'influence des élus. Elle permettait en outre de résoudre les problèmes de déficit de communication interne entre services de l'administration : le recours des administrateurs à l'influence des élus était en effet pratique courante pour dépasser les blocages internes à l'administration de l'Etat. Cette combinaison de hiérarchisation des problèmes et de résolution informelle des blocages bureaucratiques a pris le nom de « régulation croisée » (Thoenig-Dupuy 1983).

Les transformations qu'a entraînées la politique de décentralisation sont très nombreuses, à la fois pour ce qui concerne la substance du gouvernement local et dans l'évolution des concepts utilisés pour en rendre compte. Les lois de décentralisation du début des années 1980 ont consacré l'émergence de nouveaux centres de pouvoir, en conférant à des collectivités locales élues démocratiquement la responsabilité directe de compétences nombreuses : les domaines de l'urbanisme, de l'Education, du logement, de l'action sociale, de la sécurité ou de la recherche sont désormais en partie du ressort des collectivités territoriales. L'objectif de la politique de décentralisation était à la fois d'assurer une gestion plus efficace des problèmes publics, en rapprochant la décision du terrain concret de mise en oeuvre, et de démocratiser cette gestion. Sur le premier plan, c'est un modèle de répartition des compétences par blocs qui a été décidé. Le souci était d'éviter que ne se reconstituent des relations hiérarchiques de dépendance entre collectivités (communes, départements, régions). Les communes ont bénéficié de transferts dans les domaines de l'urbanisme, du logement, de l'éducation, du social. Les Conseils Généraux de département ont hérité de domaines différents : transports, éducation de premier degré, action 
sociale. Les Conseils Régionaux se sont vus conférer la responsabilité de l'éducation de second cycle, une partie de la formation professionnelle et de l'aménagement du territoire. L'Etat, qui disposait d'une tutelle a priori sur les décisions des collectivités territoriales, n'exerce plus qu'un contrôle a posteriori sur leurs actes. Cette répartition des compétences par bloc n'a certes pas toujours atteint les résultats espérés. Elle a aussi entraîné des effets inattendus : une mobilisation des collectivités locales sur leurs propres sphères de compétence ainsi que dans des domaines non décentralisés par la loi (cf. infra P.1), En ce qui concerne la seconde dimension de la décentralisation (la démocratisation), les effets de cette politique sont assez contrastés, puisque certaines des avancées incontestables (l'extension du droit de suffrage au niveau régional, le recours au scrutin proportionnel pour les élections municipales et régionales...) ont voisiné avec une portée finalement très limitée de la participation à l'action publique territoriale (cf.infra P2). $\mathrm{Au}$ delà des limites et contradictions qu'elle a engendrées, la politique de décentralisation a conduit à considérer la notion de gouvernement local avec un regard nouveau. A cette occasion, de nouvelles conceptualisations de l'action publique ont émergé, plus proches des notions débattues dans les sciences sociales anglo-saxonnes que ne l'étaient les théories françaises du système politico-administratif local ou de de la régulation croisée. Elles sont d'ailleurs souvent venues appuyer une critique de fond quant à l'adaptation des vieux outils d'analyse aux réalités nouvelles. C'est ce double parcours que nous allons suivre dans ce papier, en considérant à la fois les transformations intervenues dans la réalité du gouvernement local français et les tentatives conceptuelles d'en rendre compte. Nous nous intéresserons d'abord à l'émergence d'une pluralité d'échelles de gouvernement local, et ensuite à la dimension politique de l'action publique territoriale. Quelques considérations conclusives nous permettront de faire le bilan de l'adaptation des nouveaux concepts à une réalité française désormais moins exceptionnelle que spécifique au sein du concert européen.

\section{LES ECHELLES DU GOUVERNEMENT LOCAL FRANÇAIS :}

Le gouvernement local français, entendu comme l'ensemble des niveaux institutionnels infranationaux (l'administration déconcentrée de l'Etat et les autorités territoriales) repose sur trois échelles d'inégale importance. On compte 36763 municipalités, 100 départements (dont 4 outre- 
mer) et 26 régions (dont 21 en métropole, la Corse et 4 régions outre-mer). La caractéristique française de l'émiettement communal n'a pas été réellement modifiée. On compte ainsi 4082 communes de moins de 100 habitants, pour seulement 36 communes de plus de 100000 habitants et 5 de plus de 300000 habitants. Quant aux régions, le contraste entre les plus et les moins peuplées est très important, même en faisant abstration de la région parisienne : Rhône-Alpes, qui compte 8 départements, est peuplée de plus de cinq millions d'habitants, Provence-Alpes-Côte d'Azur en compte plus de 4 millions avec 6 départements, tandis que le Limousin (trois départements) n'en compte que 722 000, et l'Auvergne (quatre départements) 1321000 . Cette hétérogénéité démographique est une donnée importante, tant en ce qui concerne les différences de contexte sociologique du gouvernement local (cf. infra partie II) que pour les tentatives de rationnalisation de l'action publique territoriale (cf.infra plus loin).

Pour rendre compte du rôle nouveau du gouvernement local, l'un des traits majeurs réside dans l'affirmation des collectivités locales comme centres de pouvoir, associée à la pluralisation des niveaux de gouvernement. Nous allons d'abord analyser ces transformations. Elles tiennent à l'affirmation budgétaire, à l'extension des domaines d'intervention et à l'intensité comparée des ressources et capacités politiques dont disposent désormais ces différents niveaux. Elles tiennent aussi à la politique des relations entre niveaux territoriaux, dont la mise en oeuvre des politiques structurelles européennes offre un exemple caractéristique. 


\subsection{De nouvelles dynamiques dans des habits anciens}

La décentralisation française a été mise en oeuvre sans changer fondamentalement la structuration politique territoriale préexistante. Trois niveaux d'administration locale (communes, départements, régions) coexistent, qui représentent l'un des plus dense réseau d'élus et d'administrateurs au monde. C'est à celui-ci qu'ont été confiées les responsabilités politiques qui étaient auparavant du ressort de l'Etat, en fonction d'une répartition en blocs a priori rigides, mais qui se chevauchent constamment en réalité. Les compétences en matière d'éducation, qui confèrent aux communes la responsabilité de l'école primaire, aux départements celle des collèges et aux régions celle des lycées, sont très caractéristiques : aucune compétence ne peut être exercée isolément l'une de l'autre, dans un contexte marqué par des difficultés récurrentes de coordination. De même, l'action sociale, qui a très largement été confiée aux Conseils Généraux, et qui fait l'objet d'une co-gestion entre l'Etat et les départements, ne peut ignorer le fait que les communes ont été et demeurent l'un des acteurs légitimes et les plus directement sous la pression des problèmes sociaux liés aux multiples aspects de la crise économique. De fait, la pression des problèmes tend à modifier considérablement les équilibres trouvés à l'origine par le législateur français : en confiant aux départements, dès le départ, le plus important transfert de compétence, et en confirmant largement cette orientation départementaliste par la suite (mise en oeuvre du Revenu Minimum d'Insertion en 1988, du Fonds Solidarité Logement en 1992), il a ouvert la voie à un décalage important entre les compétences institutionnelles et les dynamiques territoriales (Daran 1998). Ce décalage entre les compétences officiellement transférées et celles effectivement exercées s'est généralisé. En matière de développement économique, alors que la Région était théoriquement la plus directement concernée en termes de compétences, les trois niveaux territoriales d'administrations ont rivalisé d'inventivité pour créer leur propres instruments, leurs politiques, selon une logique qui n'a pas conduit à une réelle coordination mais au contraire à une compétition et à de multiples redondances entre exécutifs (Pouvoirs locaux 1994) En matière d'action culturelle, les politiques des collectivités, peu encadrées réglementairement, ont conduit au même constat d'une compétition généralisée entre les communes, les départements et les régions. Dans cette compétition, l'Etat lui-même s'est concrètement retrouvé moins dans une position d'arbitre que de compétiteur particulier. On touche ici à une autre particularité de la décentralisation : le fait que sa dynamique n'ait pas 
seulement (et pas essentiellement) dépendu de limites réglementaires, mais au contraire d'une dynamique de conquête de responsabilités dans des domaines en partie seulement prévus par la loi. Cette dynamique a conduit à la fabrication de politiques locales dans des champs tels que la culture (Saez 1995), l'environnement (Lascoumes 1994), les télécommunications (Négrier 1996), le développement économique (Le Galès 1993), la santé (Borraz 1998b).

Même si elle a reposé sur un réseau territorial préexistant, l'extension des compétences des collectivités locales a considérablement modifié le paysage du gouvernement local. On peut repérer ces changements dans trois domaines.

\subsection{L'essor quantitatif du gouvernement local}

Le premier est l'augmentation considérable des budgets locaux, assorti d'un accroissement important du personnel des collectivités territoriales. Pour en prendre la mesure, il suffit de comparer l'évolution des dépenses communales, départementales et régionales avec celle de l'Etat.

Tableau 1 : Evolution comparée des dépenses publiques (1988-1996) (milliards de francs)

\begin{tabular}{|l|c|c|c|c|}
\hline & $\mathbf{1 9 8 8}$ & $\mathbf{1 9 9 0}$ & $\mathbf{1 9 9 3}$ & $\mathbf{1 9 9 6}$ \\
\hline Dépenses de l'Etat & 1153,6 & 1281,9 & 1502,8 & 1642 \\
\hline $\begin{array}{l}\text { Dépenses des } \\
\text { communes }\end{array}$ & 317,9 & 353,2 & 412,7 & 441,7 \\
\hline $\begin{array}{l}\text { Dépenses } \\
\text { départementales }\end{array}$ & 153,6 & 173,6 & 207,3 & 228,2 \\
\hline $\begin{array}{l}\text { Dépenses } \\
\text { régionales }\end{array}$ & 34,8 & 47,6 & 62,6 & 73,8 \\
\hline
\end{tabular}

(source : élaboration personnelle d'après Direction Générale des Collectivités Locales, Ministère de l'Intérieur 1998)

Comme on peut le voir, la progression des dépenses publiques demeure, encore aujourd'hui très forte au niveau local, plus forte en général que celle de l'Etat. On remarquera que ce sont les Régions qui ont le taux de progression le plus fort, ce qui correspond à une institution en pleine croissance, tout en étant la plus récente, puisqu'elle est issue, en tant que collectivité territoriale démocratiquement élue, des élections de 1986. Toutefois, elles ne représentent ensemble que $10 \%$ des dépenses, contre $30 \%$ pour les départements et $60 \%$ pour les communes. 
Ces données financières générales doivent être précisées par une autre indicateur : la part respective des dépenses de fonctionnement et des dépenses d'investissement des collectivités. Celui-ci traduit en effet la capacité d'initiative et d'autonomie de la collectivité : plus la part de l'investissement est important, plus cette capacité est forte. 
Tableau 2 : Part des dépenses d'investissement et de fonctionnement dans les comptes des administrations publiques (chiffres 1996) (milliards de francs)

\begin{tabular}{|l|c|c|c|c|}
\hline & Etat & Régions & Départements & Communes \\
\hline Dépense totale & 1642 & 73,8 & 228,2 & 441,7 \\
\hline Fonctionnement & 1455,5 & 30,1 & 148,6 & 306,1 \\
\hline Investissement & 186,5 & 43,7 & 79,6 & 135,7 \\
\hline $\begin{array}{l}\text { \% de dépenses } \\
\text { d'investissement }\end{array}$ & $11,35 \%$ & $59,21 \%$ & $34,88 \%$ & $30,72 \%$ \\
\hline
\end{tabular}

source : idem

On voit ici nettement que, pour être les parents pauvres de la décentralisation, les Régions sont néanmoins largement en tête pour ce qui est de leur autonomie d'action. Leurs budgets sont en effets fort peu dépendants de dépenses «obligatoires ». Contrairement aux départements, pour lesquels le transfert de compétences par l'Etat a atteint un grand volume mais en restreignant largement les choix possibles, ou aux communes, qui sont confrontées à des charges fixes assez rigides (notamment le personnel municipal), les Régions n'ont que très peu de dépenses préaffectées. La tendance est cependant à un renforcement des dépenses de fonctionnement des Régions, à la faveur des nouveaux transferts de compétence en matière de formation professionnelle.

Tableau 3 : La place de la fonction publique territoriale depuis 1969 (en milliers et en \% de la fonction publique)

\begin{tabular}{|l|l|l|l|l|l|l|l|}
\hline & $\mathbf{1 9 6 9}$ & $\mathbf{1 9 7 6}$ & $\mathbf{1 9 8 3}$ & $\mathbf{1 9 8 9}$ & $\mathbf{1 9 9 4}$ & $\mathbf{1 9 9 5}$ & $\mathbf{1 9 9 6}$ \\
\hline $\begin{array}{l}\text { Fonction publique de } \\
\text { l'Etat }\end{array}$ & 2067 & 2560 & 2813 & 2844 & 2200 & 2202 & 2214 \\
\hline $\begin{array}{l}\text { Fonction publique } \\
\text { territoriale }\end{array}$ & 618 & 859 & 1103 & 1211 & 1407 & 1428 & 1447 \\
\hline $\begin{array}{l}\text { Part de la FPT dans la } \\
\text { fonction publique (en } \\
\text { \%) }\end{array}$ & $20,4 \%$ & $21,7 \%$ & $23,6 \%$ & $24,9 \%$ & $31,7 \%$ & $32 \%$ & $32,1 \%$ \\
\hline
\end{tabular}

source: ibidem

L'importance croissante du gouvernement local se mesure également à travers l'évolution des agents des collectivités territoriales. D'une part, la part de la fonction publique territoriale dans la fonction publique a considérablement augmenté. Parallèlement, les exécutifs locaux ont recruté, par la voie des emplois contractuels, un grand nombre d'agents qualifiés, qui ont renforcé les 
capacités d'expertise et de management des autorités locales. Même si la catégorie la plus élevée de la fonction publique (catégorie A) est plus représentée dans la fonction publique d'Etat que dans la fonction publique territoriale ( $43 \%$ contre $8,6 \%)$, l'opposition entre petite et grande fonction publique tend à se réduire considérablement. 


\subsection{Centre et périphérie : vers de nouvelles interdépendances stratégiques}

Le second domaine de changement réside dans la modification des rapports centre-périphérie. Le système politico-administratif local était centré autour de l'Etat. C'était l'accès à celui-ci qui était le signe de l'importance politique des élus, et la négociation portait classiquement sur une adaptation de la règle, définie par l'Etat, à la réalité territoriale. Ce modèle a été radicalement transformé pour deux raisons.

La première tient à la pluralisation des systèmes locaux d'action publique. Celle-ci s'est traduite par l'émergence d'exécutifs forts à chacun des niveaux territoriaux. C'est par un certain paradoxe que la décentralisation, qui était censée rompre avec l'uniformité centraliste de la France jacobine, a finalement abouti à une présidentialisation de la politique locale sur un modèle très proche de la Vème République. Ce faisant, le jeu d'acteurs s'est numériquement ouvert, avec l'essor des Régions, des technostructures municipales et départementales. Dans ce nouveau système, l'Etat ne dispose plus des mêmes capacités de régulation qui étaient celles de la période centraliste. Les préfets, qui ont un temps été menacés dans leur existence même, ont certes bénéficié d'un pouvoir de coordination accru sur les services territoriaux de l'Etat (aux échelles départementale et régionale). Mais leur monopole de représentation de la règle, de l'intérêt général, et de l'expertise sur les problèmes d'action publique a disparu. Leur position d'arbitrage a donc perdu en intensité et en certitude, tandis que le pouvoir des collectivités territoriales progressait parallèlement (Kerrouche 1997).

Cette ouverture du jeu (Duran-Thoenig 1996) n'est pas seulement numérique. Elle dépend aussi de la nature des problèmes que les collectivités locales, les intérêts territoriaux et l'Etat ont à résoudre localement. La période centralisatrice de la Vème République était aussi celle de la croissance et du développement d'équipements physiques sur le territoire. Cet aménagement productif du territoire supposait le recours à des professions organisées verticalement (les Ponts et Chaussées par exemple pour les routes), qui justifiaient assez bien le recours à une expertise centralisée. La décentralisation coïncide avec la fin de cette période d'équipement qui était celle des certitudes relatives. La France des années 1990 est globalement équipée, même si quelques inégalités demeurent dans l'accès des citoyens aux routes, à l'école, à la santé...etc. La période qui s'est ouverte est celle de la crise économique et du chômage structurel, qui ne reposent ni sur les mêmes expertises concentrées, ni sur les mêmes certitudes. Du coup, la définition des problèmes elle-même fait problème. C'est ce que Patrice Duran et Jean-Claude Thoenig appellent 
le défaut de structuration des problèmes. Les enjeux deviennent de plus en plus transversaux (la politique de la ville par exemple), et l'identification des solutions repose sur une négociation de plus en plus complexe où plusieurs catégories d'intervenants (experts d'Etat, élus locaux, administrateurs territoriaux, intérêts privés...etc) disposent d’une part de légitimité.

Une grande partie des politiques qui sont mises en oeuvre sont ainsi plus procédurales que substantielles. Par politique substantielle, on entend celles dont la définition et les objectifs sont précisés a priori. Par politique procédurale, on signifie le fait que le sens et les objectifs de la politique sont au contraire très généraux, et que la fabrication réelle de son contenu est renvoyé à une délibération collective, qui se déroule à l'échelle territoriale (Lascoumes-Le Bourhis 1998). Dans cette nouvelle configuration d'acteurs et de problèmes, le rôle de l'Etat comme pôle, unique ou dominant, de définition de l'intérêt général, devient impratiquable. Ses ressources (monopole de représentation de la loi, expertise, compétences) ne lui suffisent plus pour reproduire son pouvoir et son influence sur les processus d'action publique. Il lui faut encore disposer d'une capacité à tisser les interactions stratégiques entre des acteurs à la fois légitimes et nombreux. C'est de ces interactions que découlent en effet la définition et la mise en oeuvre, a posteriori, d'un intérêt général territorialisé.

\subsection{Une gouvernement coopératif et contractuel ?}

La réalité désormais multipolaire du gouvernement local français s'exprime enfin à travers deux phénomènes relativement récents : la contractualisation et la coopération intercommunale.

Le premier aspect est une conséquence directe du processus de décentralisation, même si le lancement effectif de contrats territorialisés date des années 1970. Ces contrats, qui n’ont pas la même force juridique que les contrats de droit privé, sont l'expression d'une nécessité d'encadrement des nouveaux acteurs du gouvernement local dans des limites identifiables. Il s'agissait pour l'Etat notamment, de résister aux effets de fragmentation que pouvait engendrer la progression de la libre administration des collectivités locales. De multiples formes contractuelles ont vu le jour, parmi lesquelles on peut citer les Contrats de Plan Etat-Région, les contrats de ville, les conventions de développement culturel (Moulinier 1995) et les programmes structurels communautaires. Ceux-ci sont un bon indicateur des nouveaux rapports de force entre centre et périphérie, puisque, au-delà de la fiction juridique de l'égalité des parties au contrat, on peut 
analyser les effets de hiérarchisation des enjeux et de domination de certains acteurs. Les recherches menées sur ces nouveaux instruments de régulation centre-périphérie vont dans deux directions principales :

- la première met l'accent sur la permanence d'une influence déterminante de l'Etat dans les négociations du contenu de ces contrats. Qu'il s'agisse de leur importance en volume, des objets en négociations ou des finalités de l'action collective, la forme contractuelle aurait permis à l'Etat de conserver un rôle prépondérant dans l'action publique territoriale, tout en avançant « masqué » derrière la fabrication d'un consensus de forme. Cette démonstration a été faite pour les politiques culturelles, peu décentralisées officiellement, mais qui ont dû tenir compte du fait que les collectivités territoriales ont très tôt considéré la culture comme une catégorie stratégique d'intervention publique (Dubois \& Poirrier 1998 ; Négrier 1997b). Cette idée d'une reproduction d'une domination centrale sur la périphérie est également présente dans les travaux sur les contrats de plan Etat-Région : en établissant à environ $60 \%$ les matières que l'Etat parvient à imposer à ses partenaires, on peut voir confirmer ce rapport de force inégalitaire au profit des acteurs centraux (Cascales 1998) ${ }^{1}$.

- la second interprétation du développement de ces pratiques met au contraire l'accent sur l'ouverture des espaces de négociation, et sur les différences entre les situations d'échange, qui conduisent à des produits variables selon les territoires. C'est le coeur de certains travaux sur la décentralisation de l'art contemporain (Four 1993), ou encore de ceux qui portent sur la mise en oeuvre des politiques communautaires dans les différentes régions françaises (Smyrl 1995 ; Négrier \& Jouve 1998 ; Jouve \& Négrier 1998b, Duran 1998 ; Smith 1996). Le développement de régimes particuliers en fonction notamment de l'influence politique dont disposent les représentants des gouvernements locaux a enfin été critiquée au motif que ces régimes remettaient en question les principes, certes abstraits, mais politiquement fondés de l'égalité d'accès aux ressources publiques : la négociation de l'intérêt général sur une base territoriale et contractuelle conduirait à donner une légitimité à des intérêts particuliers, même s'ils sont exprimés par des collectivités publiques (CEPEL 1996).

Parmi les nouvelles formes contractuelles, le développement de la coopération intercommunale tient une place particulière. On sait qu'en France, le morcellement impressionnant des communes

\footnotetext{
${ }^{1}$ Cette question des rapports de force entre l'Etat et les régions a une dimension particulière à l'heure actuelle, puisque la renégociation des contrats de plan a lieu alors que trois Conseils régionaux comportent le Front National dans leur majorité.
} 
est une caractéristique historique, qui a résisté à toutes les tentatives plus ou moins autoritaires de fusion entre municipalités, dans les années 1970 et 1980. La décentralisation et l'évolution de l'occupation du territoire français posent cependant le problème d'une façon nouvelle. D'une part, en dépit des principes de décentralisation (le rejet de toute forme de tutelle hiérarchique entre collectivités), les petites communes sont rapidement devenues dépendantes, pour des raisons à la fois matérielles (leur incapacité stratégique) et politique (les réseaux localisés du cumul des mandats) des politiques menées par les Conseils Généraux. Cette nouvelle tutelle a conduit les collectivités locales à mesurer les conséquences de leur volonté traditionnelle d'autonomie vis-à-vis des bénéfices attendus des incitations publiques aux regroupements entre communes. Parallèlement à la croissance du nombre de regroupements classiques (les syndicats à vocation unique ou multiple, les districts), la loi ATR (Administration Territoriale de la République) de 1992 a défini deux nouvelles entités locales de coopération : la communauté de communes et la communauté de ville, qui ont essentiellement deux caractéristiques : la mise en commun sur leur territoire d'équipements et de services dans des domaines contractuellement définis ; 1'instauration d'une fiscalité propre, qui permet notamment une égalisation de la fiscalité à l'égard des entreprises sur la zone commune. Ces deux formes nouvelles d'intercommunalité ont la particularité de ne pas attaquer de front l'existence des communes elles-mêmes, dont l'identité politique et administrative est préservée (contrairement aux tentatives des années 1970 de fusion entre communes). En cinq ans, depuis 1992, ce sont plus de 1000 structures intercommunales qui ont vu le jour en France, regroupant plus de 10000 municipalités et 13 millions d'habitants. Parallèlement, la loi d'Aménagement et de Développement du Territoire a créé la notion de « pays », pour qualifier des bassins de vie, dont l'identité territoriale est l'une des composantes majeures, avec le fait qu'elle ne doit (théoriquement) pas coïncider avec les limites administratives existantes. Ces pays ont pour vocation de développer une « intercommunalité de projet» (Faure 1998), plus qu'une logique d'équipement du territoire. Ils connaissent actuellement un succès considérable : pour certains acteurs sociaux, ils sont le moyen de capitaliser les ressources obtenues par la mobilisation territoriale ou par la participation à certains projets de développement local (programmes Leader de l'Union Européenne par exemple) ; pour les acteurs institutionnels (les Conseils Généraux, les Conseils Régionaux), ils sont l'occasion de constituer de nouveaux relais territoriaux à leurs politiques, ou de conforter les anciens. C'est la raison pour laquelle cette forme de coopération, en cours de révision législative, 
suscite à la fois convoitises et concurrences inter-institutionnelles. On notera que les pays se situent souvent en référence à des circonscriptions antérieures à la carte administrative de la France moderne, et réhabilitent souvent des territoires de l'Ancien Régime, comme pour mieux symboliser la fin de la France jacobine.

Le gouvernement local de la France des années 1990 a inversé l'une des tendances dominantes de la politique territoriale : une action publique centrée sur l'Etat (Borraz 1998). L'émergence de nouveaux centres de pouvoir a contribué à déstabiliser le rôle de l'Etat territorial, en transformant et le fondement de son intervention légitime et les ressources stratégiques dont il dispose dans un système local désormais complexe. La distribution des pouvoirs n'a cependant pas conduit à un modèle stable de coordination, mais au contraire à des phénomènes multiples de concurrence, de redondance institutionnelle. L'inachèvement de la régionalisation, les problèmes d'adaptation des institutions communale et départementale ont généré de nouvelles recettes institutionnelles dont la stabilisation n'est pas acquise.

\section{LA DIMENSION POLITIQUE DE L'ACTION PUBLIQUE TERRITORIALE}

Le renforcement du gouvernement local français va t-il de pair avec une affirmation beaucoup plus forte qu'auparavant de la dimension politique des exécutifs locaux ? Pour s'en réjouir ou pour la craindre, c'était l'une des hypothèses fréquemment formulée dans les premières années de mise en oeuvre de cette politique. Le fait de confier à des conseils élus (et non plus à des agents de l'Etat officiellement garants d'un intérêt général abstrait) la responsabilité de domaines d'action territoriale autorise en effet à supposer que des variations importantes peuvent être constatées en fonction de la couleur politique de ces conseils. Si la question est pertinente, la réponse est pourtant beaucoup plus complexe qu'il n'y paraît. Le travail politique des élus locaux est d'abord sous la contrainte de plusieurs impératifs structurels, qui relativisent une pure et simple «politisation» des appareils locaux. Ensuite, l'observation des gouvernements territoriaux permet de mettre en évidence un processus dialectique de «différenciations territoriales » et de « standardisation politique ». Enfin, la démocratisation de l'action publique, 
qui était l'un des objectifs majeurs de la décentralisation, est sans doute l'une des dimensions les plus inachevées de ce processus.

\subsection{Les trois dimensions du travail politique local}

Le travail politique des élus est soumis à trois contraintes principales (Faure 1997). La première concerne la dimension purement élective et fait participer ces élus à une compétition politique locale qui, indépendamment des types de collectivités et de la taille des institutions, se déroule au quotidien. Un élu local français est d'abord un éligible, et cela impose un travail politique de terrain considérable. Cette première facette de l'activité politique s'observe dans la constitution des listes, lors des élections municipales et régionales, où chaque groupe social territorial, ainsi que chacun des intérêts publics et privés fait la preuve de ses propres capacités de mobilisation. L'observation de la composition des conseils municipaux en dit beaucoup sur la manière dont un chef d'exécutif est parvenu à politiser des intérêts hétérogènes et parfois divergents. Sous cet angle, l'élu est avant tout un représentant, chargé d'incarner une communauté ou des groupes potentiellement alliés ou adversaires.

La seconde dimension résulte de sa participation à un système d'action territoriale, où il enregistre et agit sur les multiples interdépendances stratégiques. Dans ce contexte, l'élu doit démontrer ses capacités à accéder aux ressources et à influencer un réseau d'acteurs qui se situe à plusieurs échelles (local, régional, national, européen) de gouvernement. La crédibilité de l'élu se joue moins à travers son statut de représentant qu'à travers une capacité à maîtriser les incertitudes liées à la complexification du système.

La troisième dimension du travail politique de l'élu est directement liée à la mise en oeuvre des politiques publiques. La négociation de l'intervention publique, la participation à des arènes (régionales ou nationales) de débat sur les orientations de politiques prennent de plus en plus d'importance : la marge de négociation s'est étendue au gré de la « procéduralisation » (cf.supra P1) de l'action publique ; la territorialisation croissante de plusieurs politiques (transports, éducation, recherche, santé, action sociale...) implique de la part des élus une mobilisation dans des domaines où l'expertise est l'une des conditions de la légitimité. C'est la raison pour laquelle les gouvernements locaux ont suivi deux tendances : 
- l'étoffement de leurs administrations, et notamment par le recours à du personnel contractuel, hors des grilles classiques de la fonction publique, ou par le transfert de personnels qualifiés de l'Etat ;

- la concession de services publics à l'initiative privée, qui, au-delà d'une tendance continentale à la privatisation, correspond aux nouvelles contraintes (normalisation, sécurité et responsabilité) d'expertise des services locaux (Lorrain 1993).

La conséquence de cette analyse des différentes dimensions du travail politique local conduit à relativiser l'importance de la politisation du gouvernement local français. Plutôt que d'avoir entraîné une politisation généralisée de l'action publique, contrairement à ce que certains avaient cru pouvoir déduire des premières années de décentralisation (Mény 1992b, Rondin 1985), celleci a au contraire conduit à relativiser l'importance de la politique sur les politiques publiques locales : celle-ci apparaît comme l'un des éléments de mise en cohérence de l'action publique territoriale, mais elle n'est ni la seule dimension à pouvoir y prétendre (vis à vis des logiques économiques, juridiques notamment) ni celle qui se distingue le plus nettement. Ce constat est valable quelque soit le sens que l'on donne à la notion de politisation : comme construction locale de projets collectifs ou comme légitimation d'orientations partisanes délibérées.

\subsection{Standardisation et nouvelles différenciations dans le gouvernement local}

Si la couleur politique des exécutifs est loin d'être un bon outil d'analyse des différences entre gouvernements locaux, on peut néanmoins identifier des divergences qualitatives et quantitatives dans la gestion locale des problèmes collectifs. Ces divergences prennent place dans un contexte de standardisation et de nouvelles logiques de différenciation territoriales.

Les tendances à la standardisation sont de trois ordres, que l'on peut illustrer pour les trois niveaux les plus évidents du gouvernement local :

- pour les villes, le facteur principal de standardisation des politiques tient à l'homogénisation relative des problèmes d'action publique, et de l'offre de biens collectifs qu'elle sous-tend : qualité des infrastructures physiques, de l'environnement, de l'offre culturelle et éducative. Ces dernières années ont ainsi consacré l'importance des grandes sociétés prestataires des services (Générale des Eaux-Vivendi ; Lyonnaise des Eaux-Dumez ; Bouygues) qui interviennent, à partir 
d'un métier principal (la distribution d'eau, le bâtiment et les travaux publics) dans un espace de plus en plus étendu de la gestion locale. Il s'agit là d'une tendance à la standardisation par l'offre de politiques publiques.

- pour les départements, la standardisation s'opère par le poids des secteurs gérés principalement par les Conseils généraux : l'aide sociale, les routes et l'aménagement du territoire. Ce sont là des domaines où la pression de la demande, le caractère obligatoire des dépenses et la standardisation des métiers imposent une tendance à la convergence des politiques.

- pour les régions, cette convergence est liée au poids que représente le co-financement par l'Etat des politiques régionales (éducation, formation, aménagement du territoire...). Le fait que les financements communautaires passent désormais par la contractualisation entre l'Etat et les Régions tend à renforcer le rôle de l'Etat comme facteur de standardisation des politiques.

Bien sûr, chaque niveau territorial demeure autonome dans un certain nombre de domaines. On peut justement expliquer le dynamisme de l'extension des compétences locales et régionales par la volonté de desserrer la contrainte de standardisation : dans la culture, dans les politiques de communication, dans les échanges internationaux, c'est à dire des domaines peu réglementés, où la décentralisation a laissé les exécutifs libres de s'investir ou non. Ceux-ci ont alors considéré ces domaines comme leurs véritable moyen de distinction et de compétition politique (Bianchini \& Parkinson 1993), même si ces nouveaux domaines ont, eux aussi, fait l'objet de formes « modernes» de standardisation, comme la contractualisation des politiques culturelles par exemple (Urfalino 1997). On peut d'ailleurs démontrer une telle tendance (sur les politiques culturelles locales) à travers le contre-exemple que représente aujourd'hui le cas des villes gérées par le Front National : la manière dont elles gèrent les questions culturelles témoigne d'une triple processus de : re-politisation, nationalisation des enjeux, de-professionnalisation des politiques : trois tendances qui s'opposent à celles qu'ont suivies la plupart des autres (dépolitisation, localisation, professionnalisation (Bressat 1999, Négrier 1997c).

Les nouvelles formes de différenciation territoriale dépendent sans doute d'autres facteurs que la simple identité politique. Plusieurs thèses s'affrontent pour qualifier ces nouvelles différences. Une première tendance, assez minoritaire en France, tente d'expliquer ces différences par la composition des réseaux d'action publique, qui sont plus ou moins inclusifs, denses et centralisés (Le Galès \& Thatcher 1995), et conduiraient à une plus ou moins grande efficience en fonction 
des scores quantitativement obtenus. Cette démarche soulève de grandes critiques en France (Hassenteufel 1995). Elle ne semble pas, en science politique, avoir dépassé le stade de l'usage métaphorique (Grote 1995, Cole\&John 1997b). Elle a été relativement peu abordée pour l'analyse du gouvernement local, même en enrichissant cette démarche par la prise en compte du contenu des interactions en réseau (Gaudin 1995)

La seconde, souvent proches des analyses en termes de gouvernance urbaine (Le Galès 1995) ou de capacité politique (Keating \& Loughlin 1996), tentent d'expliquer ces nouvelles différenciations en analysant la manière dont chaque gouvernement local parvient, ou non, à intégrer les intérêts territoriaux et à en donner une représentation unifiée à l'extérieur. Ce serait donc de la qualité des interactions ainsi orientées que naîtrait une efficience différencielle du gouvernement local, qui se traduirait à son tour par des résultats substantiellement différents. C'est ainsi que l'on pourrait notamment expliquer les différences considérables dans l'utilisation des fonds structurels par les régions françaises ${ }^{2}$, les formes divergentes de mise en oeuvre de certaines politiques sectorielles, ou encore le degré de politisation (précisément) de ces interactions stratégiques.

L'interrogation sur la qualité des interactions de type «gouvernance » n'épuise pas la signification des divergences de politiques. Elle ne parvient que difficilement à intégrer des variables historiques et culturelles qui, pour certains auteurs, sont la véritable clef de compréhension des différences entre territoires. Par exemple, certains tentent d'expliquer les forts contrastes dans la constitution de nouvelles formes de coopération intercommunale par le rôle de la culture politique territoriale : la France de l'Ouest serait ainsi plus «culturellement» disponible à la coopération, tandis que la culture politique de la France du Sud serait plus tournée vers l'autonomie égoïste et le conflit inter-institutionnel (Nevers 1998).

Cette dernière thèse fait la part belle à la reproduction dans le temps de comportement territorialement hérités. Le risque de cette forme contemporaine de culturalisme est connu, et renvoie par exemple aux critiques émise à l'encontre des thèses de R.D.Putnam. Les tendances au changement, visibles dans l'ensemble du territoire français, contredisent tous les jours le prétendu

\footnotetext{
${ }^{2}$ Aujourd'hui, une grande majorité de régions françaises sont contraintes à rembourser les sommes affectées à leur territoire par l'Union Européenne dans le cadre des fonds structurels (Midi-Pyrénées, Centre, Languedoc-Roussillon, Nord-Pas de Calais). Quelques unes d'entre elles (Haute-Normandie, Poitou-Charentes, Alsace), cependant, ont à tel point consommé leurs enveloppes qu'elles sont candidates à une augmentation de leur dotation (Négrier 1998b).
} 
effet de variables culturelles aussi «dures ». On militera ici pour une tentative de conciliation entre une perspective synchronique qui ôte toute épaisseur à l'Histoire et une perspective diachronique qui lui accorde trop de crédit. Si l'on s'accorde à constater que les nouvelles formes d'interdépendance territoriale ne sont pas nécessairement toutes innovantes, qu'elles associent des acteurs (souvent les mêmes) dont la coopération et les conflits se produisent et se reproduisent dans le temps, on peut alors parler d'échange politique territorialisé (Négrier 1998 c), pour prendre en compte à la fois le degré d'historicité des comportements d'interaction (la reproduction de « configurations d'échanges ») et l'évolution des scènes d'échange par l'effet des apprentissages dont les acteurs qui participent à ces scènes bénéficient. Cette dialectique du changement et des reproductions de configurations territoriales désigne en même temps les effets de réduction de la participation effective à l'action publique, et rejoint en cela les interrogations en termes de démocratie locale.

\subsection{Les transformations de la démocratie locale}

L'un des objectifs poursuivis par les inspirateurs de la décentralisation était précisément celui d'approfondir la démocratie française. Confisquée par une élite centralisée, peu représentative de la population dans toutes ses nuances, la démocratie représentative trouverait dans la sphère locale les racines d'une régénération. Quinze ans plus tard, on parle davantage de monarchie municipale que de république locale (Mabileau 1995). La démocratie locale est fréquemment considérée comme le plus grand échec de la décentralisation. Si certains éléments peuvent être effectivement mis au crédit d'une démocratisation de la vie publique, on va voir que celle-ci demeure limitée dans ses effets pratiques.

Parmi les facteurs de démocratisation du gouvernement local, on distinguera ici trois processus : la localisation de la décision, l'extension du gouvernement représentatif et la limitation du cumul des mandats.

La localisation de la décision est un acquis de la décentralisation. Tous les processus que nous avons précédemment décrits vont dans le sens d'une réappropriation par les sociétés locales des enjeux qui concernent leurs territoire. La notion d'affaire locale, qui était très sévèrement limitée par la jurisprudence administrative, est désormais extensive, à la fois par les compétences réellement déléguées et à travers les dynamiques d'échange politique territorialisé. Ceci dit, cette 
localisation va de pair avec la persistance d'une fermeture relative des cercles d'action légitime. On peut ilustrer ce constat par deux exemples : la participation des associations au pouvoir local et la mise en oeuvre du partenariat local. Sur le premier plan, on constate que les relations entre associations et municipalités sont structurellement déséquilibrées au profit des secondes, qui ont, à travers la manipulation de la ressource financière et politique, une réelle capacité à s'imposer sans réel contre-pouvoir au sein de la société civile (Balme 1987). Les associations qui parviennent à acquérir un certain poids sont souvent celles qui ont choisi de s'aligner plus ou moins sur les orientations majoritaires, en troquant l'impératif d'adhésion contre le désir d'influence. Certaines d'entre elles parviennent toutefois à maintenir une autonomie en jouant sur les compétitions entre niveaux d'action publique : le contre-pouvoir local exercé par une association doit alors être interprété comme un alignement de plus haut degré territorial. Quant au partenariat local, si souvent consacré comme le principe d'action publique des temps modernes, il est bien souvent à la recherche d'autres partenaires que les simples autorités publiques. La faible légitimité dont jouissent en France les intérêts privés organisés localement continue, malgré les incantations (publiques), à limiter le contenu et les effets du partenariat public-privé. La démocratie locale, entendue comme une extension des processus de participation à l'action publique, est de ce fait largement théorique. La multiplication des procédures de consultation, plus ou moins formelle, de la population ne fait pas obstacle à ce que le gouvernement par la délibération ne progresse guère (Piéchaczyk 1998, Blatrix 1996).

L'extension du gouvernement représentatif local est le deuxième aspect de la démocratie locale. Elle s'est traduite par deux phénomènes observables : l'extension du suffrage universel à de nouvelles assemblées et la proportionnalisation d'une partie de la vie politique. Sur le premier point, l'approfondissement démocratique est manifeste : les français ont désormais la possibilité de désigner démocratiquement leurs conseillers municipaux, leurs conseillers généraux et leurs conseillers régionaux, en plus des élections présidentielles, parlementaires et européennes. Sur le second aspect, l'introduction du mode de scrutin proportionnel pour les élections municipales et régionales s'est traduit par l'arrivée au sein des exécutifs locaux d'une opposition institutionnalisée. Elle est également à l'origine d'un établissement politique du Front National. Mais la progression de la représentativité n'est pas généralisée, et ses effets sont paradoxaux.

Tout d'abord, on assiste au maintien de modes de scrutin locaux en complet décalage avec l'évolution de la population. C'est en particulier le cas de l'élection des Conseils Généraux, qui 
reposent sur la base de cantons où dominent fortement les espaces ruraux. La conséquence en est que l'échelon le plus puissant du gouvernement local est en même temps celui qui repose sur la base démocratique (et démographique) la plus contestable.

Ensuite, les nouvelles échelles du gouvernement local comme la région ne sont pas, de manière évidente, légitimées par le personnel politique lui-même. Les conseils régionaux sont encore, après trois mandatures, des espaces politiques subsidiaires, où l'élection siginifie plus une rétribution militante ou clientéliste qu'un véritable engagement politique sélectif (Nay 1998). La forte rotation du personnel politique régional, si on le compare, par exemple, avec celui des Communautés autonomes espagnoles est un autre indice de cette faible régionalisation du système politique français (Négrier $1998 \mathrm{~d}$ ).

Enfin, la présence d'une opposition institutionnalisée au sein des conseils municipaux et régionaux ne s'est pas traduite par une réelle intensification du débat public local. Au contraire, les gouvernements municipaux ont eu tendance à priver l'opposition des moyens d'information stratégique, en concentrant ceux-ci au sein de cabinets toujours plus étoffés. En conséquence, un décalage de plus en plus fort s'est établi entre la sphère de l'action (les sommets de l'administration et le chef de l'exécutif) et la sphère de la représentation (les conseils élus). La présidentialisation de la première va de pair avec la mise à distance de la seconde. L'opposition joue d'autant moins son rôle politique qu'elle entend en général appliquer le même cloisonnement en parvenant à son tour au pouvoir. Il est frappant de constater que parmi les affaires qui ont secoué les villes et pouvoirs locaux ces dernières années, aucune n'a pour origine la dénonciation de l'opposition : elles ont en revanche souvent pour point de départ l'action isolée d'un citoyen bien informé et tenace, ou bien les investigations d'un juge.

La réduction du cumul des mandats est typique de la volonté de mettre fin à une exception française. Ses difficultés de mise en oeuvre sont symboliques de l'enracinement de ce type de pratique politique. Contrairement à une idée souvent diffusée, le cumul des mandats (la possibilité de détenir à la fois un mandat de parlementaire, de chef d'exécutif local et régional, de député européen...) ne remonte pas à très loin dans l'histoire. La généralisation de sa pratique ne s'est en effet produite qu'à l'occasion de la Vème République ${ }^{3}$. Elle s'est renforcée à l'occasion de la décentralisation, alors que, paradoxalement, croissaient l'importance et l'intensité du travail 
politique local. On peut expliquer ce paradoxe par les fonctions que remplit un tel cumul (Alliès 1998) :

- une facilitation des réélections, pour un personnel politique qui augmente ses chances parallèlement à son accaparement des mandats ;

- une rationalisation des niveaux de décision territorialisée, dont les logiques de fragmentation sont parfois opaques. Elles trouvent dans le cumul une forme de personnalisation et de concrétisation des enjeux politiques.

- une légitimation territoriale accrue dans les interdépendances stratégiques (avec l'administration tout comme avec les intérêts privés).

Mais le cumul des mandats est l'un des obstacles de fait à l'instauration d'un véritable pluralisme démocratique local, pour trois raisons :

- il concentre les caractéristiques des élites locales sur des types limités. Le faible renouvellement sociologique y trouve sa source : $20 \%$ des maires sont agriculteurs, alors que ceux-ci ne représentent que 4,6\% du corps électoral ; pour les maires urbains, ce sont quelques professions qui dominent selon le même décalage : professions libérales; cadres supérieurs et moyens, patrons (Garraud 1989). Cette concentration sociale vaut également pour la sous-représentation des femmes $(10 \%$ des conseillers régionaux, $5 \%$ des conseillers généraux, $21 \%$ des conseillers municipaux mais seulement $7,5 \%$ des maires). Ce constat est d'autant plus important qu'il concerne une population d'élus très nombreuse : 544000 élus, soit dix fois plus qu'en GrandeBretagne).

- il conduit à des inégalités de prise en compte des intérêts locaux en fonction de l'existence ou non de plusieurs mandats exercés puisque l'accès aux ressources est facilité par le cumul, et l'emporte sur d'autres considérations plus objectives (des besoins par exemple).

- il dépolitise l'exercice du pouvoir, en conduisant par exemple à un absentéisme parlementaire structurel : la participation des parlementaires à l'activité législative (travail en Commission, enquête, débats) est en effet contradictoire avec les exigences de la multi-positionnalité politique (le travail de terrain l'emporte souvent puisqu'il assure la véritable continuité de la carrière). La dépolitisation se traduit aussi par la perte de pouvoir des partis politiques sur des élus locaux fortement enracinés. Même si le cumul, une première fois limité (par la loi de 1985, cf Mény

\footnotetext{
${ }^{3}$ La proportion de députés détenant plusieurs mandats n'était que de 49\% en 1958. Elle est passée, selon une progression linéaire, à 96\% en 1988 et se stabilise aujourd'hui à 93\% (Alliès 1998).
} 
1997), est aujourd'hui à nouveau visé par des projets de réduction ${ }^{4}$, la capacité du personnel politique à en contourner les effets est enfin très forte. Le fait que la première loi ait (ironiquement) beaucoup fait pour la féminisation des élites locales (les épouses des élus cumulant remplaçant leurs maris !) dit assez bien la résistance d'un tel phénomène social et politique.

\section{CONCLUSION :}

Le gouvernement local français connaît des facteurs importants de stabilisation : du personnel politique, des élites dirigeantes, des processus de professionnalisation, de dépolitisation, et de localisation des enjeux. Ils ont pour contrepartie la récurrence des problèmes de coordination (ou d'intégration des différents niveaux), de démocratisation (au double sens d'ouverture de la représentation (parité homme-femmes, origines socio-professionnelles...) et d'ouverture de la participation à l'action publique : déficit de partenariat public-privé, faiblesse de l'évaluation...

Sur le plan des outils d'analyse, le bouillonnement dont les sciences sociales ont fait preuve depuis dix ans, en tentant souvent d'ailleurs d'acclimater des concepts anglo-saxons, a permis de préciser où se situaient les insuffisances des conceptualisations antérieures ( en termes de système politico-administratif local ou de régulation croisée), et d'atteindre à un type d'analyse désormais ouvert au comparatisme. On doit cependant reconnaître que ces concepts se heurtent à des difficultés de plusieurs ordres. Par exemple, l'analyse des réseaux ne va pas au delà d'une utilisation métaphorique, et conduit à une impasse d'ordre méthodologique ; une certaine conception de la gouvernance (la plus prescriptive) se heurte à l'absence des éléments empiriques nécessaires (comme l'indique la faiblesse du partenariat) à étayer la démonstration ; les notions de contractualisation, de négociation sont des outils de description de processus, mais difficiles à transformer en concepts plus généraux, de même sans doute que l'échange politique. Dans le champ désormais pluraliste de la recherche française sur le gouvernement local, on doit enfin noter que certaines pistes, qui pouvaient paraître prometteuses, sont inachevées, abandonnées ou s'autonomisent de façon dommageable : l'anthropologie du pouvoir local n'a guère survécu aux travaux de Marc Abelès (1986, 1989); les analyses historiques du pouvoir local (Dumons-Pollet-

\footnotetext{
${ }^{4}$ La loi du 30 décembre 1985 a rendu le mandat de député ou de sénateur incompatible avec plus d'un des mandats suivants : député européen, conseiller régional, conseiller général, maire d'une commune de plus de 20000 habitants
} 
Saunier 1997) sont peu prises en compte par les analystes contemporains. Enfin, la faiblesse historique des intérêts privés organisés territorialement n'a d'égal que celle des travaux qui lui sont consacrés : au delà des perspectives fonctionnalistes (sur la bonne gouvernance et ses best practices), cet approfondissement sera l'un des enjeux d'un renouvellement de l'analyse politique du gouvernement local.

\section{References :}

Abelès, Marc (1986) : «Le degré zéro de la politique. Réseaux de pouvoir et espace intercommunal dans le canton de Quarré-les-Tombes (Morvan) » in Etudes Rurales n 101-102, Paris, Laboratoire d'Anthropologie Sociale, pp.231-271

Abelès, Marc (1989) : « Jours tranquilles en 1989 » Odile Jacob, Paris

Alliès, Paul (1998) : «Les effets du cumul des mandats sur le personnel politique », in CREAM : «Le cumul des mandats et des fonctions. Une réforme au coeur de la modernisation de la vie politique » La Documentation Française, Paris, pp.63-76

Balme, Richard (1987) : « La participation aux associations et le pouvoir municipal. Capacités et limites de la mobilisation par les associations culturelles dans les communes de banlieue », Revue Française de Sociologie, Paris pp. 601-639

Bianchini, Franco \& Parkinson, Michael (eds) (1993) : « Cultural Policy and Urban Regeneration. The West European Experience » Manchester University Press

Blatrix, Cécile (1996) : «Vers une démocratie participative ? Le cas de l'enquête publique » in in CURAPP : La gouvernabilité » Presses Universitaires de France, Paris. pp.299-314

Borraz, Olivier (1998) : « Pour une sociologie des dynamiques de l'action publique locale » in Balme, Richard, Faure, Alain and Mabileau, Albert : «Politiques locales et transformations de l'action publique en Europe » CERAT, Grenoble, pp.85-101

Borraz, Olivier (1998b) : « Les politiques locales de lutte contre le SIDA. Une analyse dans trois départements français » L'Harmattan, Logiques Politiques, Paris (avec Patricia Loncle-Moriceau et Christel Arrouet)

Bressat, Cécile (1999) : «Culture et autorité partisane : la politique de « rééquilibrage de la bibliothèque d'une municipalité FN. Orange 1995-1997) Pôle Sud n¹0, Montpellier pp.75-92

Cascales, Michèle (1998) : « Le contrat de Plan Etat-Région est-il un outil de programmation pluri-annuelle? » Revue Politique et Parlementaire n 995, Paris Juillet, pp.93-102 
CEPEL (1996) : « La négociation des politiques contractuelles » L’Harmattan, Logiques Politiques Paris

Cole, Alistair \& John, Peter (1997a) : "Capacity building, networks and local political leadership" in Gerry Stoker (ed.) The new management of local governance : Audit of an Era of Change in Local Governance" London:Macmillan 1997

Cole, Alistair \& John, Peter (1997b): "Networks or networking ? The importance of power, position and values in local economic policy networks in Britain and France" Paper for APSA Conference 1997, 28-31 august Washington USA

Daran, Michèle (1998) : "L'action sociale des communes. Un secteur de contrastes » in Balme, Richard, Faure, Alain and Mabileau, Albert : « Politiques locales et transformations de l'action publique en Europe » CERAT, Grenoble, pp.157-168

Direction Générale des Collectivités Locales (1998) : «Les collectivités locales en chiffres » Ministère de l'Intérieur, La Documentation Française, Paris

Dubois, Vincent \& Poirrier Philippe, eds (1998) : « Politiques locales et enjeux culturels. Les clochers d'une querelle XIXème-XXème siècle » Documentation Française Paris

Dumons, Bruno - Pollet, Gilles - Saunier, Pierre-Yves (1997) : « Pouvoirs locaux et élites municipales. Analyse comparée des villes de la France du Sud-Est » Editions du CNRS, Paris

Duran, Patrice (1998) : « Le partenariat dans la gestion des fonds structurels : la situation française » Pôle Sud n8, Montpellier pp.114-140

Duran, Patrice \& Thoenig Jean-Claude (1996) : «L'Etat et la gestion publique territoriale » Revue Française de Science Politique vol 46 n², pp. 580-623

Faure, Alain (1997) : «Les apprentissages du métier d'élu local. La tribu, le système et les arènes » Pôle Sud nº 7, Montpellier, pp.72-80

Faure, Alain (1998) : «L'intercommunalité de projet, un modèle d'action publique innovant ? » Observatoire sur l'Intercommunalité de Projet en Rhône-Alpes, Rapport 50 p., CERAT-IEP de Grenoble

Four, Pierre-Alain (1993) : «La compétence contre la décentralisation ? Création des Fonds Régionaux d'Art Contemporain » Politix n²4, L’Harmattan Paris pp.95-114

Garraud, Philippe (1989) : «Profession : homme politique. La carrière politique des maires urbains » L'Harmattan, Paris

Gaudin, Jean-Pierre (1995) : «Politiques urbaines et négociations territoriales : quelle légitimité pour les réseaux de politiques publiques ? » Revue Française de Science Politique n45 (1) pp.3156 
Grémion, Pierre (1976) : Le pouvoir périphérique. Bureaucrates et notables dans le système politico-administratif français » Le Seuil, Paris

Grote, Jurgen (1995) : « Réseaux de politique publique ou échange clientéliste : des métaphores à l'évaluation des relations Etat-société » Pôle Sud n³, Montpellier, pp.55-71

Hassenteufel, Patrick (1995) : « Do policy networks matter ? Lifting descriptif et analyse de l'Etat en interaction » in Le Galès, Patrick \& Thatcher, Mark (1995) : "Les réseaux de politique publique » L'Harmattan, Logiques Politiques, Paris, pp.91-109

Jouve, Bernard (1995) : «Réseaux et communautés de politique publique en action » in Le Galès, Patrick \& Thatcher, Mark (1995) : «Les réseaux de politique publique » L'Harmattan, Logiques Politiques, Paris, pp.121-141

Jouve, Bernard \& Négrier, Emmanuel (1998) : « Multi-Level Governance « French Style »? The Contribution of the European Structural Funds to Redefining Intergovernmental Relations in France » European Planning Studies, Vol.6, n5, pp.555-572

Keating, Michael, Loughlin John (eds) (1996) : « The Political Economy of Regionalism », Frank Cass, London

Kerrouche, Éric (1997) : «L'apprentissage du rôle de sous-préfet » Politix n³8, L’Harmattan Paris pp.88-110

Lascoumes, Pierre - Le Bourhis Jean-Pierre (1998) : « Le bien commun comme construit territorial. Identité d'action et procédures », Politix n²4, L’Harmattan Paris pp.37-66

Lascoumes, Pierre (1994) : «L’éco-pouvoir. Environnement et politiques » La Découverte Paris

Le Galès, Patrick (1993) : « Politique urbaine et développement local » L’Harmattan Paris

Le Galès, Patrick (1995) : « Du gouvernement des villes à la gouvernance urbaine » Revue Française de Science Politique ${ }^{\circ} 45$ (1) Paris pp.57-95

Le Galès, Patrick \& Thatcher, Mark (1995) : « Les réseaux de politique publique » L'Harmattan, Logiques Politiques, Paris

Lorrain, Dominique (1993) : «Après la décentralisation, l'action publique flexible », Sociologie du Travail n³, Paris pp. 285-307

Mabileau, Albert (1995) : «De la monarchie municipale à la française » in Pouvoirs $n^{\circ} 73$ « La démocratie municipale », Le Seuil, Paris, pp.7-18

Mény, Yves (1992b) : « La corruption de la République » Fayard, Paris

Meny, Yves (1997) : «Le cumul républicain : la démocratie réduite aux acquets? » Revue Politique et Parlementaire ${ }^{\circ} 991$ Paris, pp.5-12 
Moulinier, Pierre (1995) : «Politique culturelle et décentralisation » Editions du Centre National de la Fonction Publique Territoriale, Paris

Nay, Olivier (1998) : «La région, une institution. La représentation, le pouvoir et la règle dans l'espace régional » L'Harmattan, Logiques politiques Paris

Négrier, Emmanuel (1997a) : « Espaces urbains et sociétés de communication » Espaces et Sociétés n87, L’Harmattan Paris 1996, pp.59-91

Négrier, Emmanuel (1997b) : «French Cultural decentralization and International Expansion » International Journal of Urban and Regional Research vol.21 $\mathrm{n}^{\circ} 1$, Blackwell Publishers Oxford

Négrier, Emmanuel (1997c) : « The professionnalisation of urban cultural policies : the case of festivals » Government \& Policy 1996, vol 14, pp.515-529

Négrier, Emmanuel \& Jouve, Bernard (eds) (1998) : «Que gouvernent les Régions d'Europe ?» L'Harmattan, Logiques Politiques Paris

Négrier 1998b) : « The Thematic Assessment of the Partnership Principle in the Structural Funds. The case of France » Report for Tavistock Institute-ECOTEC and the European Commission, Birmingham-Bruxelles

Négrier, Emmanuel (1998 c) : « Que gouvernent les Régions d’Europe ? Introduction » L'Harmattan, Logiques Politiques Paris pp.11-31

Négrier, Emmanuel (1998d) : «Une action publique sans coopération politique : le style languedocien de politique régionale »Pôle $\mathrm{Sud}^{\circ}{ }^{\circ}$, Montpellier pp.41-54

Nevers, Jean-Yves (1998) : «La relance de l'intercommunalité. Contextes et stratégies » in Balme, Richard, Faure, Alain and Mabileau, Albert : " Politiques locales et transformations de 1'action publique en Europe » CERAT, Grenoble, pp.221-231

Piechaczyk, Xavier (1998) : « Le rôle des commissaires-enquêteurs et l'intérêt général » in Politix n42, Paris, pp.93-122

Pouvoirs locaux (1994) : « Poursuivre la décentralisation. Réflexions sur le bilan et les perspectives de la décentralisation » Editions Pouvoirs locaux, Paris

Rangeon, François (1996) : Le gouvernement local» in CURAPP : La gouvernabilité » Presses Universitaires de France, Paris. pp.166-174

Rondin, Jacques (1985) : « Le sacre des notables » Fayard, Paris

Saez, Guy (1995) : «Villes et culture : un gouvernement par la coopération » Pouvoirs $n^{\circ} 73$, PUF Paris pp. 109-125 
Schmidt, Vivien (1990) : Democratizing France : The Political and Administrative History of Decentralization » New-York, Cambridge University Press

Smith, Andy (1996) : « Putting the Governance back into Multilevel Governance. Examples from French Translations of the Structural Funds » paper for European Consortium for Political Research, Olslo

Smyrl, Marc (1995) : «From regional policy to European networks : interregional divergence in the implementation of the EC Structural Funds in France » European University Institute, Firenze, Working Paper

Thoenig, Jean-Claude \& Dupuy, François (1983) : « Sociologie de l'administration» Colin, Paris Urfalino, Philippe (1997) : «L’invention de la politique culturelle » Documentation Française, Paris

Waters, Sarah (1998) : "Chambers of Commerce and Local Development in France : problems and constraints" Government and Policy vol.16, London pp.591-604

Worms, Jean-Pierre (1966) : « Le préfet et ses notables »Revue Française de sociologie, 3, Paris pp. $255-260$ 